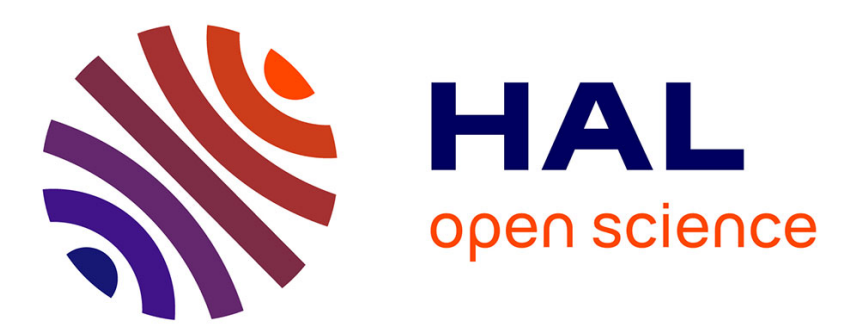

\title{
Analyse de données ellipsométriques provenant de l'investigation de films très minces absorbants et homogènes
}

\author{
B. Agius, J. Siejka
}

\section{- To cite this version:}

B. Agius, J. Siejka. Analyse de données ellipsométriques provenant de l'investigation de films très minces absorbants et homogènes. Revue de Physique Appliquée, 1977, 12 (8), pp.1171-1180. 10.1051/rphysap:019770012080117100 . jpa-00244295

\section{HAL Id: jpa-00244295 https://hal.science/jpa-00244295}

Submitted on 1 Jan 1977

HAL is a multi-disciplinary open access archive for the deposit and dissemination of scientific research documents, whether they are published or not. The documents may come from teaching and research institutions in France or abroad, or from public or private research centers.
L'archive ouverte pluridisciplinaire HAL, est destinée au dépôt et à la diffusion de documents scientifiques de niveau recherche, publiés ou non, émanant des établissements d'enseignement et de recherche français ou étrangers, des laboratoires publics ou privés. 
Classification

Physics Abstracts

8.360

\title{
ANALYSE DE DONNÉES ELLIPSOMÉTRIQUES PROVENANT DE L'INVESTIGATION DE FILMS TRÈS MINCES ABSORBANTS ET HOMOGËNES (*)
}

\author{
B. AGIUS et J. SIEJKA \\ Groupe de Physique des Solides de l'Ecole Normale Supérieure, \\ Tour 23, 2, Place Jussieu, 75221 Paris Cedex 05, France
}

(Reçu le 30 novembre 1976, accepté le 20 avril 1977)

\begin{abstract}
Résumé. - Nous décrivons un procédé d'obtention de films d'oxyde de fer, minces, homogènes, absorbants, d'épaisseur bien déterminée inférieure à $30 \AA$. La stoechiométrie de ces films formés par oxydation anodique d'un substrat de fer a été déterminée par utilisation d' 180 et par observation d'interactions nucléaires induites par des faisceaux d'ions légers monocinétiques d'une énergie voisine $\mathrm{du} \mathrm{MeV}$. L'analyse détaillée des résultats provenant d'une investigation ellipsométrique de ces films est présentée : nous montrons que même dans le cas d'une étude de films homogènes, l'ellipsométrie ne permet pas de déduire les constantes optiques des films ; elle donne cependant l'ordre de grandeur de leur épaisseur. Par contre l'association de ces résultats à ceux obtenus par microanalyse nucléaire nous a permis de déterminer les constantes optiques de ces films très minces, absorbants.
\end{abstract}

\begin{abstract}
A new process is described for obtaining homogeneous, absorbing iron oxide films with well-defined thicknesses below $30 \AA$. The stoichiometry of these films which were formed by anodic oxidation of an iron substrate was determined by using ${ }^{18} \mathrm{O}$ and by observing the nuclear interactions induced by monokinetic light ion beams with energies around $1 \mathrm{MeV}$. A detailed analysis of the results of an ellipsometric study of these films is presented : it shows that the optical constants of these films cannot be determined by ellipsometric methods only. It shows, however, that the order of magnitude of film thicknesses can be deduced. By combining these results with those of nuclear microanalysis, it was possible to determine the optical constants of these ultra-thin absorbing films.
\end{abstract}

1. Introduction. - Les très nombreuses techniques utilisées pour l'étude des surfaces sont toutes limitées par la nature des informations qu'elles peuvent fournir. Parmi ces méthodes, l'ellipsométrie est de plus en plus pratiquée en tant que méthode non destructive permettant de travailler in situ. Elle semble adaptée à l'investigation de films très minces $(d<100 \AA)$ formés aussi bien en solution aqueuse - films anodiques et passifs - que déposés sur un substrat dans une atmosphère sous vide. Elle utilise un faisceau incident d'énergie lumineuse assez faible pour ne pas perturber la surface du film ou l'interface film-substrat contrairement aux méthodes d'analyse nécessitant l'utilisation de faisceaux d'ions.

Elle est extrêmement sensible : Archer a détecté par ellipsométrie une monocouche d'oxygène adsorbé sur du silicium [1] ; Melmed et al. [2] ont également montré que l'adsorpion d'une monocouche d'oxygène sur du tungstène $(001)$ est aisément détectable et

(*) Les travaux présentés dans cet article ont été effectués dans le cadre de la R. C. P. $n^{\circ} 157$ du C. N. R. S. Ces travaux ont aussi reçu le soutien de la D. G. R.S.T. et de la D. R. M.E. conduit à des variations importantes des paramètres ellipsométriques. En tant que méthode d'analyse qualitative, elle permet donc de suivre toute évolution d'une surface, de mettre en évidence une adsorption quelconque se situant à la surface du métal supposé exempt de tout film superficiel, de suivre l'évolution d'un film formé par la variation de ses propriétés optiques etc..

En tant que méthode d'analyse quantitative, elle permet de déterminer $a$ priori l'épaisseur et les constantes optiques de films épais homogènes [3-4]. Dans le cas d'étude de films minces absorbants, son utilisation est très fréquente [5-8] bien que l'interprétation quantitative des résultats soit très controversée [9] : en effet, pour déterminer les constantes optiques d'un film absorbant ainsi que son épaisseur, nous disposons habituellement à une longueur d'onde donnée des deux seuls paramètres expérimentaux $\Delta$ (retard relatif de phase) et $\Psi$ (tg $\Psi$ étant le rapport d'amplitude relative). Ceux-ci devront donc êrre utilisés pour calculer trois inconnues : $n_{\mathrm{F}}$ et $k_{\mathrm{F}}$, parties réelle et imaginaire de l'indice de réfraction du film étudié, et $d$, épaisseur de ce film. Une multitude de solutions possi- 
bles sera obtenue, ce qui diminue grandement l'intérêt de l'utilisation de l'ellipsométrie en tant que méthode d'investigation de films absorbants. Habituellement, cette indétermination est levée soit en déterminant préalablement l'épaisseur par une autre méthode d'analyse, indépendante de l'ellipsométrie (par exemple, détermination de l'épaisseur par coulométrie ou par microanalyse nucléaire pour des films minces formés anodiquement) soit en essayant de connaître les constantes $n_{\mathrm{F}}$ et $k_{\mathrm{F}}$ du film. Nous discutons en détail ces deux possibilités dans le paragraphe suivant, en présentant les avantages mais surtout les inconvénients relatifs à leur application dans l'étude de films très minces.

Le but de ce travail est d'insister sur les difficultés rencontrées lors d'une investigation purement ellipsométrique de couches très minces, homogènes, absorbantes, d'épaisseur connue. Nous venons en effet de préciser que si l'ellipsométrie est largement utilisée dans l'étude de tels films, l'interprétation des résultats reste très discutée pour les raisons suivantes :

- cette méthode expérimentale ne fournit aucune information sur l'homogénéité d'un film mince absorbant,

- en outre, elle ne donne qu'un ordre de grandeur de son épaisseur.

Par l'étude de l'homogénéité d'un film, nous enten- dons l'étude de l'ensemble des connaissances liées à sa structure atomique ainsi qu'à sa composition. Cette homogénéité est usuellement reliée à la constante de l'indice optique de ce film étudié à différentes épaisseurs. En ce sens, l'homogénéité physique d'un film peut être assimilée à son homogénéité optique (constance de $n_{\mathrm{F}}^{*}=n_{\mathrm{F}}-i k_{\mathrm{F}}$ ). En outre, nous montrerons que même associée à une autre méthode qui permettrait de connaître l'épaisseur géométrique équivalente du film étudié, l'ellipsométrie ne fournirait qu'un ordre de grandeur de l'indice optique le caractérisant; en effet, ce dernier serait connu avec une précision directement reliée à l'erreur entachant la mesure d'épaisseur qui, dans les cas les plus favorables, serait au moins égale à $5 \%$. L'indice optique serait alors déterminé avec une imprécision telle qu'il deviendrait impossible d'en vérifier la constance pour des films de différentes épaisseurs et donc d'en déduire leur homogénéité optique et par là même leur homogénéité physique.

2. Dispositif expérimental. - L'instrument utilisé est un appareil Rudolph, modèle 437-200 E légèrement modifié. Sa configuration est montrée (Fig. 1) ; les différents composants de l'ellipsomètre ont été placés de telle sorte que le polariseur et la lame quart-d'onde soient situés avant la réflexion de la lumière par la surface cristalline. Le polariseur et l'analyseur sont des prismes Glan-Thomson dont les rotations autour

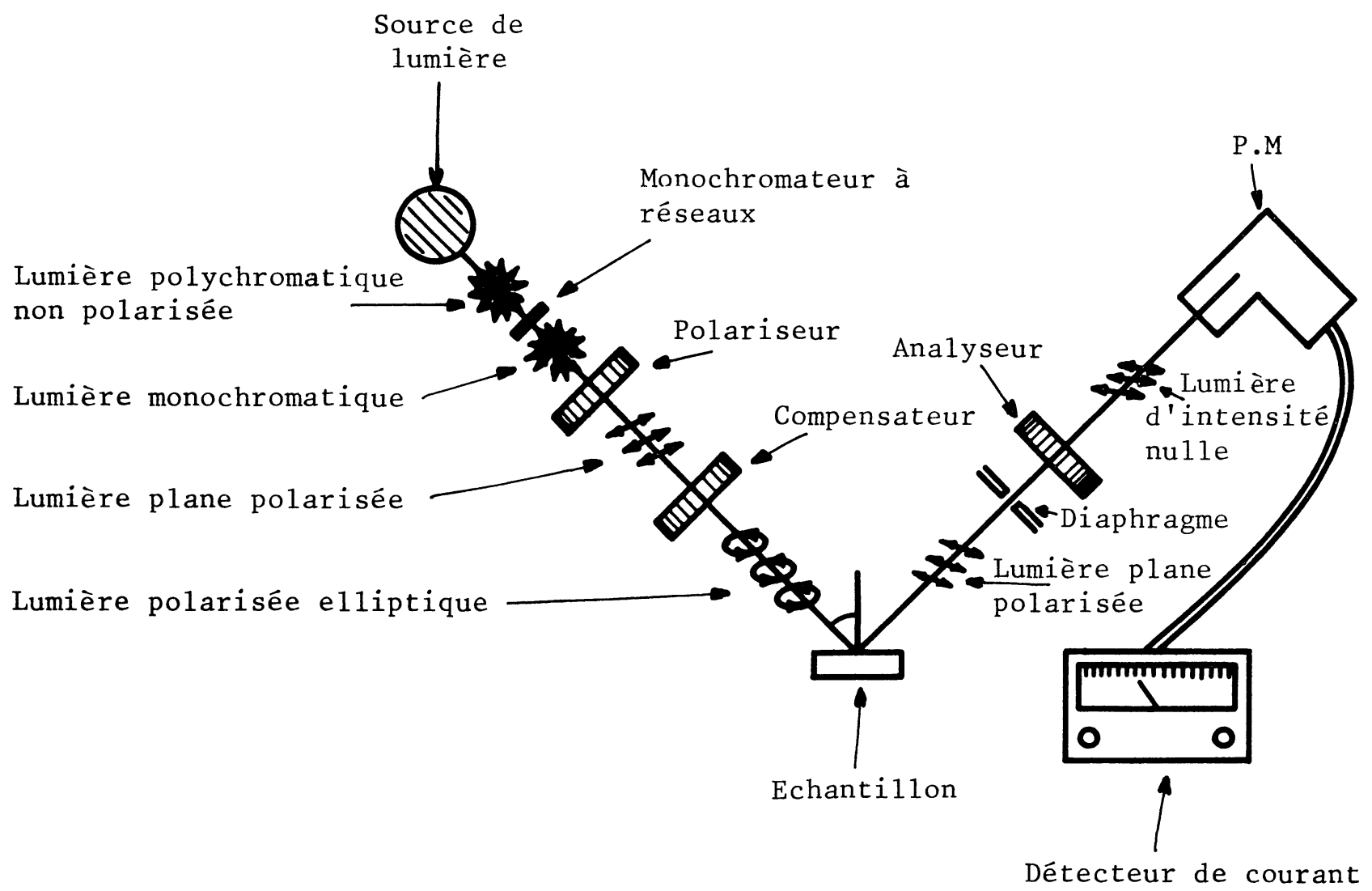

FIG. 1. - Les différents composants de l'ellipsomètre utilisé

[Block diagram of the instrument.] 
d'un axe perpendiculaire à leurs faces sont lues à $0,01^{\circ}$ près. L'alignement de l'instrument s'effectue par rapport à l'échantillon et suivant la procédure décrite par Mac Crackin et al. [10]; le retard relatif de phase (4) ainsi que le rapport d'amplitude relatif $(\operatorname{tg} \Psi)$ des deux composantes parallèle et perpendiculaire du faisceau réfléchi sont alors déterminés. Le signal détecté par un photomultiplicateur est ensuite enregistré. La précision obtenue sur la détermination de $\Delta$ et de $\psi$ est alors d'environ $0,03^{\circ}$. Une optimalisation des conditions de détection est actuellement à l'étude et porte à la fois sur une diminution du courant d'obscurité du photomultiplicateur utilisé et sur l'utilisation d'une lumière modulée.

Toutes les mesures ellipsométriques ont été effectuées à un angle d'incidence de $70^{\circ} 36^{\prime}$ et pour différentes longueurs d'onde comprise entre 4000 et $8000 \AA$. Pour chaque longueur d'onde, le déphasage $\Delta_{\mathrm{c}}$ et $1 \mathrm{e}$ coefficient de transmission $T_{\mathrm{c}}$ entre les vibrations transmises respectivement par les axes rapide et lent de la lame quart d'onde ont été calculés [11]. Le programme utilisé détermine systématiquement l'erreur introduite par le compensateur et y apporte les corrections appropriées.

3. Obtention et caractérisation de couches minces absorbantes, homogènes, d'épaisseur déterminée. Nous décrivons ci-dessous la formation d'un film mince homogène absorbant, d'épaisseur bien déterminée comprise entre 0 et $30 \AA$; il s'agit d'un film passif d'oxyde de fer formé dans une solution tampon de $0,15 \mathrm{~N}$ de borate de sodium et $0,15 \mathrm{~N}$ d'acide borique, contenant $4 \times 10^{-4}$ mole/litre de $\mathrm{As}_{2} \mathrm{O}_{3}$ et maintenue à $-3^{\circ} \mathrm{C}$. La cellule utilisée permettant d'effectuer des mesures optiques et nucléaires a été décrite précédemment [12]. Le processus de formation du film passif a par ailleurs été largement mentionné $[12,13]$; nous en rappelons ici les principales étapes :

3.1 ProcéDÉ eXPérimental. - Après polissage de l'échantillon de fer $(99,99 \%)$ [13], celui-ci est réduit cathodiquement, $\varphi_{\mathrm{c}}=-650 \mathrm{mV}(\mathrm{H}$. E.), afin d'éliminer tout film superficiel présent à la surface. Le prétraitement cathodique terminé, la polarisation est stoppée 5 minutes, procédé qui améliore la reproductibilité des films. Une augmentation du potentiel est alors observée, de $\varphi_{\mathrm{c}}=-650 \mathrm{mV}$ (H. E) à $\varphi_{\mathrm{s} 2}=-510 \mathrm{mV}$ (H. E). Ce phénomène est probablement relié à l'expulsion de l'hydrogène pouvant être présent au voisinage de la surface du métal. Puis un potentiel positif est appliqué à l'échantillon par l'intermédiaire d'un potentiostat.

3.2 MISE EN ÉVIDENCE DE L'HOMOGÉNÉITÉ DU FILM. Nous avons précédemment montré [12] en utilisant la technique de traçage à $1^{\prime} \mathrm{O}^{18}\left({ }^{1}\right)-$ technique abon-

(1) L'eau enrichie en 180 est produite à l'Institut WeizmannRehovot (Israël). damment décrite par ailleurs $[14,15,16,17]-$ qu'un film d'oxyde de fer formé à potentiel élevé, $+940 \mathrm{mV}$ (H. E.), dans une telle solution, se réduisait de façon homogène avec un rendement faradique de $100 \%$ suivant le mécanisme $(a)$ :

$$
\begin{array}{r}
\mathrm{Fe}_{2} \mathrm{O}_{3(\text { oxyde) }}+2 \mathrm{e}^{-}+4 \mathrm{H}^{+} \rightarrow 2 \mathrm{FeOH}_{\text {(solution) }}^{+}+ \\
+\mathrm{H}_{2} \mathrm{O}
\end{array}
$$

Ce mécanisme associe $2 / 3$ de charge électronique élémentaire à un atome d'oxygène passant en solution.

Il est illustré sur la figure 2 par la ligne continue $(a)$. Celle-ci se trouve directement confondue avec la

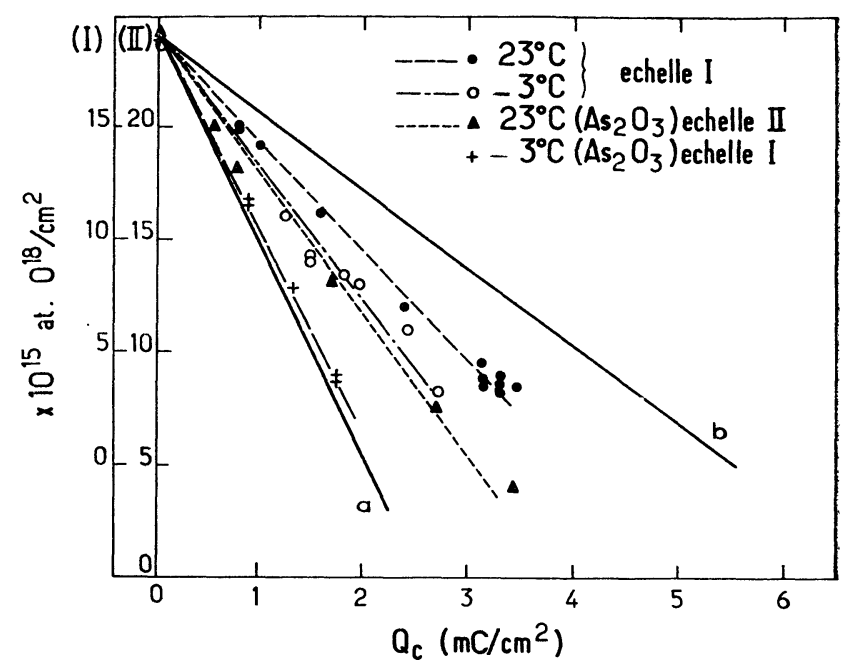

Fig. 2. - Variation de la quantité d'O18 lors de la réduction cathodique à $10 \mu \mathrm{A} / \mathrm{cm}^{2}$ d'un film passif de fer formé potentiostatiquement en $3 \mathrm{~min}$. $\left(I_{\text {final }}=10 \mu \mathrm{A} / \mathrm{cm}^{2}\right)$ à $+940 \mathrm{mV}$ et à différentes températures. Le film est formé dans une solution neutre de $0,15 \mathrm{~N}$ de borate de $\mathrm{Na}$ et d'acide borique [13].

[Change in $\mathrm{O}^{18}$ content of iron during galvanostatic cathodic reduction at $10 \mu \mathrm{A} / \mathrm{cm}^{2}$ for the passive films formed potentiostatically at $+940 \mathrm{mV}$ in a $0.15 \mathrm{~N}$ boric-borate buffer solution [13]]

droite expérimentale $(+)$ donnant la variation de la quantité d'oxygène ${ }^{18} \mathrm{O}$-mesurée aux moyens de la réaction nucléaire ${ }^{18} \mathrm{O}(\mathrm{p}, \alpha)^{15} \mathrm{~N}$-fixée à la surface d'un échantillon au cours de sa réduction cathodique. Les deux droites sont confondues: cela implique donc que :

- la composante oxyde de fer du film passif formé dans ces conditions expérimentales (application d'un champ électrique très élevé pendant un temps très court) a bien pour stoechiométrie $\mathrm{Fe}_{2} \mathrm{O}_{3}$.

- qu'il s'agit d'un oxyde compact.

- que sa réduction s'effectue de façon homogène.

En effet tout autre composé associerait une charge élémentaire $n$ différente pour chaque atome d'oxygène passant en solution :

- si la formule du composé se rapprochait de $\mathrm{Fe}_{3} \mathrm{O}_{4}[7,18]$ la réduction s'effectuerait suivant

$$
\mathrm{Fe}_{3} \mathrm{O}_{4}+2 \mathrm{e}^{-}+8 \mathrm{H}^{+} \rightarrow 3 \mathrm{Fe}^{2+}+4 \mathrm{H}_{2} \mathrm{O}
$$


soit

$$
n=0,5 \mathrm{e}
$$

- si la stœchiométrie était $\mathrm{Fe}^{6+} \mathrm{O}_{3}$ [18], le processus serait

$$
\mathrm{Fe}^{6+} \mathrm{O}_{3}+4 \mathrm{e}^{-}+5 \mathrm{H}^{+} \rightarrow \mathrm{Fe}^{2+} \mathrm{OH}^{+}+2 \mathrm{H}_{2} \mathrm{O}
$$

soit

$$
n=\frac{4}{3} \mathrm{e} .
$$

Ainsi, seule une réduction s'effectuant d'après le mécanisme (a) apporte un ensemble important d'informations susceptibles de caractériser de façon univoque le film mince étudié, à savoir son homogénéité, sa structure et sa stoechiométrie. Il convient de remarquer à ce stade que seule la microanalyse nucléaire par utilisation d'isotopes stables de l'oxygène - films formés dans des solutions enrichies en ${ }^{18} \mathrm{O}$ - permet de mesurer directement la charge élémentaire associée à chaque atome d'oxygène passant en solution. Les autres méthodes, qu'elles soient électriques ou optiques, la déduisent à partir d'hypothèses plus ou moins discutables.

L'existence d'un rendement faradique de réduction cathodique égal à $100 \%$ indique l'inhibition de tout dégagement $\mathrm{d}$ 'hydrogène et de toute réduction des ions ferriques $\mathrm{Fe}^{3+}$ à l'état métallique $\mathrm{Fe}^{0}$. La réduction $\mathrm{Fe}^{3+} \rightarrow \mathrm{Fe}^{0}$ a notamment été observée par Sato et al. [7] pour des films passifs de fer, formés cependant dans des conditions expérimentales différentes. L'existence d'un rendement faradique de réduction cathodique égal à $100 \%$ peut être attribué à la présence d'arsenic incorporé à l'intérieur de l'oxyde lors de sa formation, étant donné le rôle bien connu d'inhibiteur de corrosion de cet élément. Une telle présence a été confirmée et chiffrée [12] : elle est de $2 \times 10^{15}$ atomes $/ \mathrm{cm}^{2}$ d'arsenic pour un film formé à $+940 \mathrm{mV}$ (H. E.). La connaissance simultanée des quantités d'oxygène $\left(18 \times 10^{15}\right.$ at $\left.{ }^{18} \mathrm{O} / \mathrm{cm}^{2}\right)$ et d'arsenic $\left(2 \times 10^{15}\right.$ at $\left.{ }^{75} \mathrm{As} / \mathrm{cm}^{2}\right)$ présents dans le film permettent d'en déduire la stæchiométrie du composé, soit :

$$
\left(\mathrm{Fe}_{2} \mathrm{O}_{3}\right)_{4,66}\left(\mathrm{As}_{2} \mathrm{O}_{5}\right)
$$

pour un oxyde formé à $+940 \mathrm{mV}$ (H. E.).

Le même raisonnement et les mêmes mesures effectuées pour plusieurs films réduits cathodiquement pendant différents temps conduisent à la détermination de la même stoechiométrie du composé.

3.3 ESTIMATION DE L'ÉPAISSEUR GÉOMÉTRIQUE ÉQUIVALENTE. - La stoechiométrie du film étant connue, nous avons calculé sa densité en appliquant une simple loi additive aux densités des composés séparés $\mathrm{Fe}_{2} \mathrm{O}_{3}$ et $\mathrm{As}_{2} \mathrm{O}_{5}$. Nous avons ainsi trouvé $\rho=5,0 \mathrm{~g} / \mathrm{cm}^{3}$. Pour cette densité, l'épaisseur d'une couche contenant $10^{15}$ at $\mathrm{O} / \mathrm{cm}^{2}$ est équivalente à $1,7 \AA$. Nous sommes donc en mesure de calculer maintenant l'épaisseur d'un film formé à $+940 \mathrm{mV}$ (H. E.) puis réduit cathodiquement à différentes épaisseurs après avoir vérifié la structure - compacte et homogène - du composé et déterminé sa stœechiométrie : $\left(\mathrm{Fe}_{2} \mathrm{O}_{3}\right)_{4,66}\left(\mathrm{As}_{2} \mathrm{O}_{5}\right)$.

Quel type d'informations peut-on alors obtenir par une investigation purement ellipsométrique de ces films formés in situ, d'épaisseur et de stœchiométrie contrôlée et bien déterminée ?

4. Etudes ellipsométriques de films minces, homogènes et compacts. - A chaque couple de valeurs $P$ et $A$ déterminées pour une position azimutale $\mathrm{Q}$ du compensateur correspondra un ensemble de valeurs de l'indice de réfraction complexe du film et de son épaisseur ; ceci suppose connus l'indice de réfraction du substrat ainsi que celui de la solution utilisés lors de l'étude de la structure métal-solution. Suivant le processus de calcul habituellement utilisé [19], nous calculons les épaisseurs $d$ et les constantes optiques du film

$$
n_{\mathrm{F}}^{*}=n_{\mathrm{F}}-i k_{\mathrm{F}}
$$

en supposant $n_{\mathrm{F}}$ et $k_{\mathrm{F}}$ variant entre un minimum et un maximum. Ces trois grandeurs sont en effet déterminées en faisant varier l'indice d'absorption $k_{\mathrm{F}}$ du film de sa valeur initiale imposée à sa valeur finale par addition successive d'un incrément $\delta K_{\mathrm{F}}$. Pour chaque coefficient d'absorption choisi, $\Delta$ et $\psi$ sont calculés et comparés à $\Delta$ et $\psi$ mesurés ; si l'écart entre ces résultats reste compris à l'intérieur d'une certaine marge d'erreur admise, $d$ et $n_{\mathrm{F}}^{*}$ représentent alors une des solutions recherchées. Dans le cas contraire, il n'y a pas de solution pour cette valeur du coefficient d'absorption et le processus est répété pour la valeur suivante de $k_{\mathrm{F}}$. La limite d'erreur a été fixée à $0,02^{\circ}$, cette valeur se rapprochant de celle propre aux conditions expérimentales qui sont les nôtres dans le cas de surfaces immergées.

Le choix des valeurs de ces différents paramètres joue un rôle fondamental pour l'obtention des solutions. Il doit être judicieux pour ne pas risquer d'éliminer une solution possible [20].

4.1 EVAluation Des PaRAMÈTRes OPTIQUeS A PARTIR DE MESURES ELLIPSOMÉTRIQUES : RÉSULTATS ET INTERPRÉTATION. - Les mesures ellipsométriques sont en général sensibles à de nombreux facteurs: la rugosité de la surface, la pureté de la solution, la pureté du métal. Elles doivent être effectuées avec grand soin pour être reproductibles. L'un des problèmes fondamentaux pour l'étude ellipsométrique de films superficiels est la nature du substrat puisque ce dernier est en général utilisé comme référence optique lorsqu'il est exempt de tout film superficiel [21].

4.1.1. Détermination des constantes optiques du métal pur. - Il est donc important de connaître les constantes optiques du substrat utilisé sur lequel sera formé le film passif. En effet, une faible variation de 
l'indice de réfraction du substrat entraîne des variations importantes des grandeurs $n_{\mathrm{F}}$ et $k_{\mathrm{F}}$ qui caractérisent le film étudié. Sur le tableau I, nous représentons les valeurs d'indice obtenues pour un film d'oxyde de

\section{TABLEAU I}

Comparaison des constantes optiques d'un film obtenues pour différentes valeurs de substrat. Les valeurs de $\lambda, 5461 \AA$; de l'angle d'incidence, $70^{\circ} 60$; de l'épaisseur, $39 \AA$ sont fixées. $\psi_{\text {mesuré }}=27,79^{\circ}$; $\Delta_{\text {mesuré }}=103,80^{\circ}$

$$
N_{\mathrm{s}}
$$

Travail actuel [12]

$3,30(1-1,12 i)$

$3,31(1-1,14 i)$

$3,35(1-1,14 i)$

Kruger (1969) [9]

$3,18(1-1,21 i)$

Sato et Kudo (1971) [7]

$3,24(1-1,24 i)$

Bockris (1971) [8]

fer, d'épaisseur supposée connue (39 ̊), pour différentes valeurs de l'indice optique du substrat. De l'observation de ces résultats, il ressort qu'il n'est pas raisonnable d'essayer de déterminer les constantes optiques d'un film mince $(d<100 \AA)$ sans avoir auparavant mesuré les caractéristiques optiques du substrat sur lequel il est formé. On ne doit pas, à partir d'un couple de valeurs mesurées $P$ et $A$ obtenu sur une structure substrat-film, déduire les constantes optiques du film à partir de celles déterminées par un autre auteur. Qu'y a-t-il de commun entre les deux solutions

$$
n_{\mathrm{F}}^{*}=2,60(1-0,05 i)
$$

et

$$
n_{\mathrm{F}}^{*}=3,20(1-0,25 i)
$$

obtenues pour le même couple de valeurs mesurées $\psi$ et $\Delta$ mais à partir de deux substrats, la première obtenue par nous-mêmes [12] :

$N_{\mathrm{S}}=(3,3 \pm 0,2)-(3,7 \pm 0,2) i$, la seconde donnée par Bockris [8] $N_{\mathrm{s}}=(3,24 \pm 0,2)-(4,61 \pm 0,2) i$.

Comme on ne peut jamais être sûr de l'état d'une surface immergée dans une solution aqueuse, même lorsque cette dernière est maintenue sous polarisation cathodique, la meilleure approche est de comparer les constantes optiques ainsi obtenues avec celles connues pour la même surface étudiée en ultra-vide. C'est ce que nous avons fait en comparant (Fig. 3) l'indice de réfraction d'un échantillon de fer réduit cathodique-
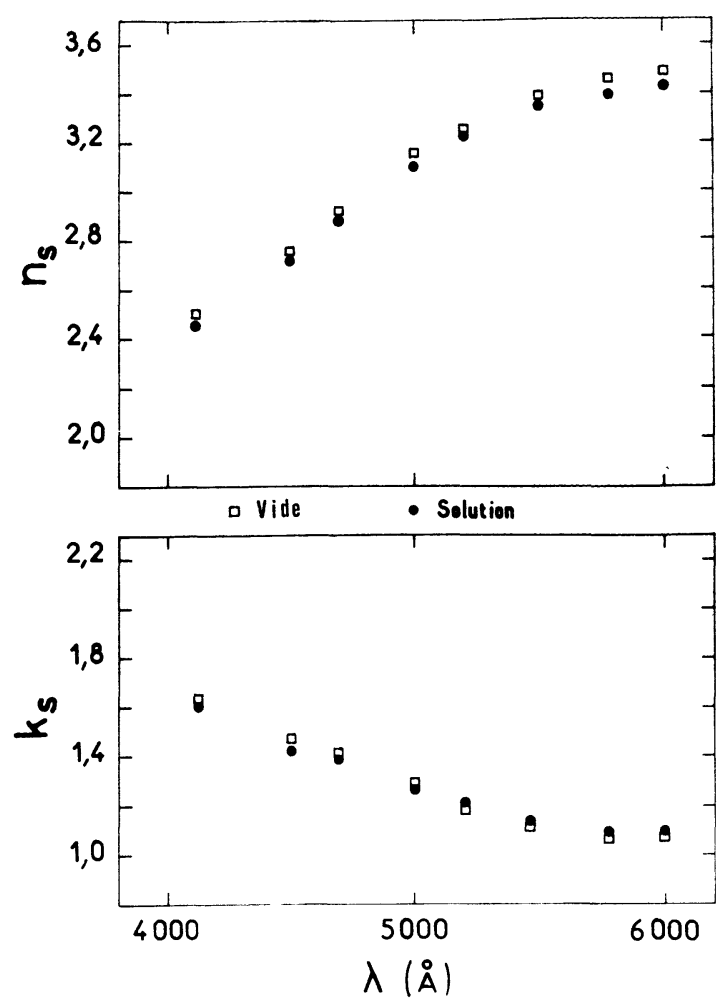

Fig. 3. - Comparaison des constantes optiques d'une surface de fer dépourvue d'oxyde naturel : $\square$ obtenue sous vide par ellipsométrie [9]; $\bigcirc$ polarisée cathodiquement dans une solution tampon ( $\mathrm{pH}=8,42)$ constituée d'un mélange égal de $0,15 \mathrm{~N}$ de borate de sodium et $0,15 \mathrm{~N}$ d'acide borique.

[Comparison of the optical constants of a vacuum-produced clean Fe surface [9] and a surface reduced cathodically in $0.15 \mathrm{~N}$ boric-borate buffer solution.]

ment [13] $\left(I=10 \mu \mathrm{A} / \mathrm{cm}^{2}\right)$ dans une solution de borate, avec celui obtenu par Kruger sur une surface de fer maintenue en ultra-vide et sur laquelle le L. E. E. D. a montré l'absence de tout corps adsorbé [9].

La bonne correspondance entre ces deux figures est considérée comme critère de pureté pour les surfaces étudiées en solution aqueuse, maintenues sous polarisation cathodique. A titre d'exemple, nous avons représenté (Fig. 3) la variation de l'indice du substrat $\mathrm{du}$ fer en fonction de la longueur d'onde. Nous avons déterminé le spectre optique de films minces dans une gamme de longueur d'onde variant entre $4000 \AA$ et $8000 \AA$ et étudié en particulier la variation de $n_{\mathrm{F}}$ et $k_{\mathrm{F}}$ avec la longueur d'onde. L'investigation de films passifs par ellipsométrie spectroscopique pourrait être une voie permettant de résoudre le problème des solutions multiples des équations ellipsométriques appliquées aux films absorbants. Ce point fera l'objet d'une prochaine publication, mais nous pouvons déjà préciser que cette impression est fausse dans le domaine de longueurs d'onde généralement étudié.

4.1.2. Essai de détermination des constantes optiques et de l'épaisseur d'un film mince absorbant à partir d'une investigation purement ellipsométrique. - Après 
avoir arrêté l'oxydation anodique à courant constant $\left(5 \mu \mathrm{A} / \mathrm{cm}^{2}\right)$, les valeurs azimutales du polarisateur $P$ et de l'analyseur $A$, donnant l'extinction, sont recherchées puis notées. La figure ( $4 a$ ) donne la variation de $\Delta P$ en fonction du potentiel tandis que la figure (4b) illustre la variation correspondante de $A$ en fonction de $P$. La grandeur $\Delta P$ est comptée à partir de la valeur azimutale de $P$ obtenue sur l'électrode supposée exempte de tout film superficiel ; sa variation linéaire avec le potentiel semble indiquer que le film formé est composé d'une seule et même couche qui pourrait cependant ne pas être homogène à l'échelle atomique.

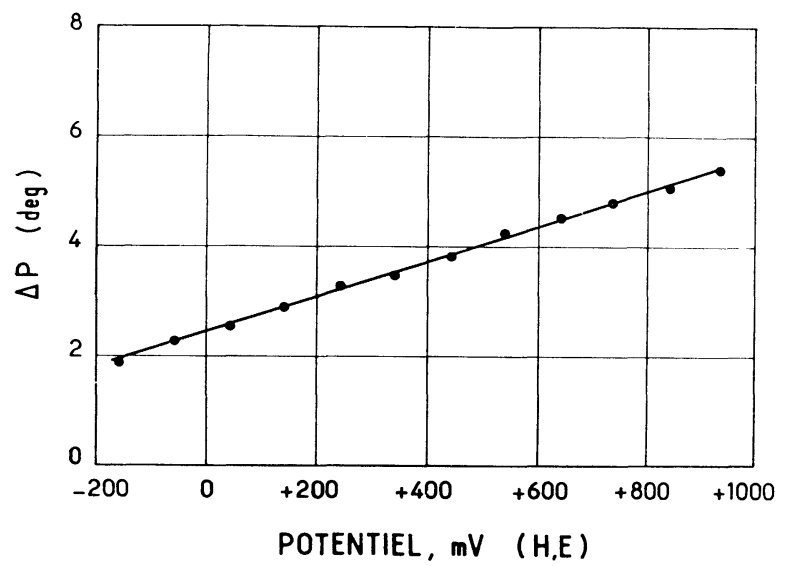

(a)

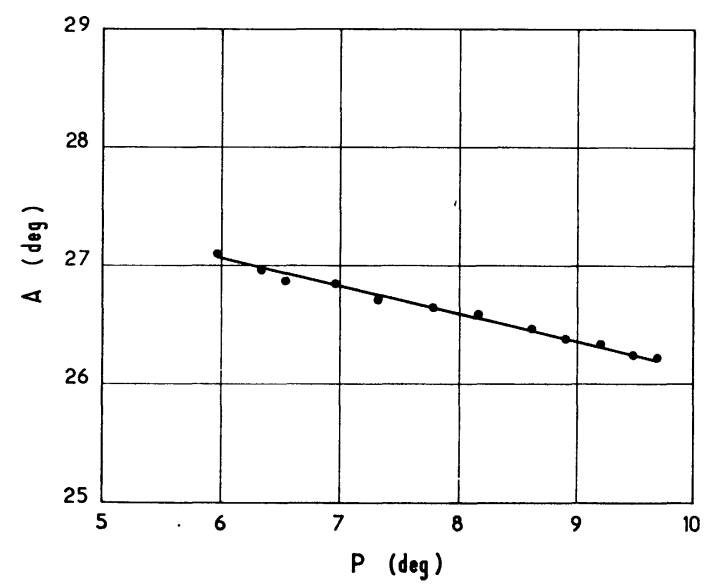

(b)

Fig. 4. $-(a)$ Variation de $-\Delta P$ pour un échantillon de fer oxydé potentiostatiquement $\left(I_{\text {final }}=5 \mu \mathrm{A} / \mathrm{cm}^{2}\right)$. Même solution que pour la figure 2. (b) Variation de $A$ en fonction de $P$ pour un échantillon de fer oxydé potentiostatiquement $\left(I_{\text {final }}=5 \mu \mathrm{A} / \mathrm{cm}^{2}\right)$ Même solution que pour la figure 2 .

[a) Change in ellipsometric parameters $-\Delta P$ for the passive films formed potentiostatically. The solution was the same as for figure 2. b) Change in ellipsometric parameters $P$ and $A$ for the passive films formed potentiostatically. The solution was the same as for figure 2.]

Pour chaque valeur de $P$ et $A$ mesurés (Fig. $4 b$ ) nous pouvons calculer, comme nous l'avons indiqué pré- cédemment, une multitude de solutions possibles pour les parties réelle $\left(n_{\mathrm{F}}\right)$ et imaginaire $\left(k_{\mathrm{F}}\right)$ de l'indice de réfraction du film et pour son épaisseur $(d)$ (Fig. 5).

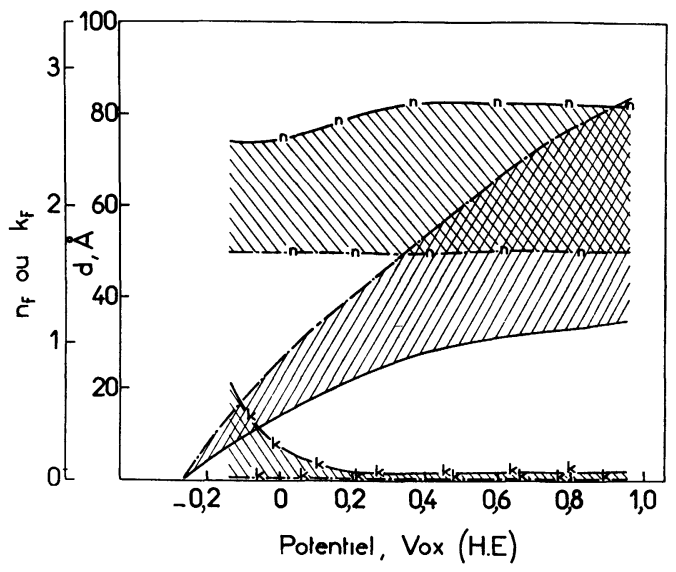

FIG. 5. - Variation de $d, n_{\mathrm{F}}$ et $k_{\mathrm{F}}$ en fonction du potentiel pour un échantillon de fer oxydé potentiostatiquement [12] $\left(I_{\text {final }}=5 \mu \mathrm{A} / \mathrm{cm}^{2}\right)$ lorsque les constantes optiques $n_{\mathrm{F}}$ et $k_{\mathrm{F}}$ du film sont supposées variées dans les intervalles 1-3,5 et 0-1. Même solution que pour la figure 2 .

[Change of $d, n_{\mathrm{F}}$ and $k_{\mathrm{F}}$ of passive iron films formed potentiostatically [12] $\left(I_{\text {rinal }}=5 \mu \mathrm{A} / \mathrm{cm}^{2}\right)$ with the optical constants $n_{\mathrm{F}}$ and $K_{\mathrm{F}}$ assumed to be in the ranges 1-3.5 and 0-1. The solution was the same as for figure 2.]

Remarquons que l'incertitude sur la détermination de l'épaisseur du film augmente avec le potentiel : de 20 à $\pm 5 \AA$ à $\mathrm{OV}$, elle passe à $60 \pm 20 \AA$ à $950 \mathrm{mV}$. Seul un ordre de grandeur de l'épaisseur peut donc être retenu. Par contre aucune valeur de l'indice optique ne peut être déduit de cette investigation.

Si l'on désire déterminer l'épaisseur avec une précision plus grande, il faut, auparavant, déterminer les constantes optiques du film, et vice-versa ; si l'on veut connaître les constantes optiques du film, il faut déterminer préalablement son épaisseur. Une troisième voie consisterait à augmenter le nombre de mesures ellipsométriques indépendantes les unes des autres en faisant varier par exemple :

- l'angle d'incidence,

- les épaisseurs du film étudié s'il est homogène,

- le milieu environnant,

cette approche devant permettre de définir le troisième paramètre expérimental nécessaire à la détermination des trois inconnues que sont $n_{\mathrm{F}}, k_{\mathrm{F}}$ et $d$.

4.1.2.1. Augmentation du nombre de mesures ellipsométriques indépendantes. - a) Variation de l'angle d'incidence. - A première vue cette approche semble très intéressante. Elle est souvent présentée et utilisée par différents auteurs comme l'une des possibilités les plus sérieuses susceptibles de rendre l'ellipsométrie self consistante [22-23]. L'analyse des récentes données de la littérature met en évidence deux cas possibles d'études suivant la valeur de l'angle d'incidence retenu. 
- Pour des angles $\varphi$ très faibles, Gottesfeld [24], faisant intervenir la constante diélectrique complexe du matériau plutôt que son indice optique, a présenté les équations de Drude [25] sous la forme d'expressions simplifiées

$$
\begin{aligned}
\delta \Delta \simeq-(4 \pi d / \lambda) \sqrt{\varepsilon}, \cos \varphi & \operatorname{tg}^{2} \varphi \times \\
\times & \operatorname{Re}\left[\left(\hat{\varepsilon_{2}}-\hat{\varepsilon}_{3}\right) /\left(\varepsilon_{1}-\hat{\varepsilon}_{3}\right)\right]
\end{aligned}
$$

et

$$
\begin{aligned}
& \delta \psi \simeq(2 d / \lambda) \sin ^{2} \psi \sqrt{\varepsilon}, \cos \varphi \operatorname{tg}^{2} \varphi \times \\
& \times \operatorname{Im}\left[\left(\hat{\varepsilon}_{2}-\widehat{\varepsilon}_{3}\right) /\left(\varepsilon_{1}-\widehat{\varepsilon}_{3}\right)\right]
\end{aligned}
$$

$\varepsilon_{1} \widehat{\varepsilon}_{2}$ et $\hat{\varepsilon}_{3}$ étant respectivement les constantes diélectriques du milieu environnant, du film et du substrat.

L'analyse de ces équations permet de conclure :

- qu'elles ne sont ni linéaires ni homogènes (vis-àvis des trois variables indépendantes $d, n_{\mathrm{F}}, k_{\mathrm{F}}$ ),

- que le fait de changer l'angle d'incidence n'ajoute aucune nouvelle équation indépendante aux deux précédentes puisque $\varphi$ intervient comme facteur multiplicatif dans ces deux équations.

- Pour les angles d'incidence habituellement utilisés en ellipsométrie $\left(\varphi \simeq 70^{\circ}\right)$, le problème semble plus complexe. La solution dépend en effet des épaisseurs de films étudiés et des précisions entâchant les mesures expérimentales. Schueller [26] a notamment montré que, dans les conditions habituelles d'expérience $\left(\delta \Delta=\delta \psi=0,01^{\circ}\right)$, l'analysc des résultats obtenus pour trois angles d'incidence $\left(\varphi_{\mathbf{i}}=60^{\circ}, 70^{\circ}\right.$, $80^{\circ}$ ) conduit à une solution unique pour des films dont l'épaisseur $d$ est supérieure à $200 \AA$, mais à la détermination de différents triplets $d$, $n_{\mathrm{F}}$ et $k_{\mathrm{F}}$ pour $d \ll \lambda$ $(d \simeq 50 \AA)$.

Il semble cependant possible dans le cadre d'étude de films très minces d'aboutir à une solution unique [22-23] si la précision des mesures est largement améliorée pour atteindre le millième de degré $\left(\delta \Delta=\delta \psi=\delta \varphi=0,001^{\circ}\right)$. Ceci suppose de s'affranchir de différentes contraintes expérimentales et impose en particulier un choix d'échantillons à surface parfaitement lisse.

b) Variation de l'épaisseur du film. - Un ensemble de mesures peut être effectué sur des films de différentes épaisseurs à condition de s'être assurés auparavant de l'homogénéité de ces films. Dans ce seul cas bien précis, une comparaison entre les différents résultats obtenus laisse apparaître à toutes les épaisseurs du film étudié, des valeurs communes pour l'indice optique, ce qui permet d'éliminer les valeurs extrêmes des constantes optiques. Il faut cependant noter que cette approche ne convient qu'à des films d'épaisseur supérieure à $50 \AA$. En effet, les remarques précédemment effectuées à partir de la dépendance de $\Delta$ et $\psi$ en fonction du paramètre $\varphi$ sont également valables lorsque ce paramètre est l'épaisseur du film $d$. Ceci signifie donc que la variation de l'épaisseur $d$ d'un film ne peut usuellement constituer une possibilité de rendre l'ellipsométrie self consistante lorsque cette dernière est appliquée à l'investigation de films minces inférieurs à $50 \AA$. Nous avons confirmé expérimentalement cette observation, basée sur l'interprétation d'équations dérivant de celles de Drude et développées au premier ordre en appliquant cette technique aux couches minces précédemment décrites d'épaisseur déterminée (comprise entre $36 \AA$ et $2 \AA$ ) et dont l'homogénéité avait par ailleurs été établie. Aucune solution unique pour l'indice optique de ces couches n'a pu être mise en évidence : nous en avons représenté

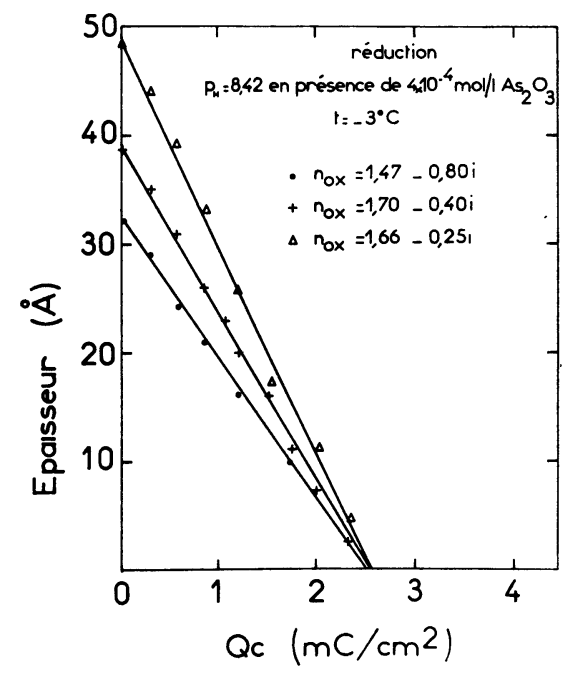

Fig. 6. - Variations de l'épaisseur lors de la réduction cathodique à $10 \mu \mathrm{A} / \mathrm{cm}^{2}$ d'un film passif de fer formé potentiostatiquement en $3 \mathrm{mn}\left(I_{\text {final }}=10 \mu \mathrm{A} / \mathrm{cm}^{2}\right)$ à $+940 \mathrm{mV}$. Les constantes optiques $n_{\mathrm{F}}$ et $k_{\mathrm{F}}$ du film sont supposées variées dans les intervalles 1-3,5 et 0-1. Même solution que pour la figure 2 .

[Change of film thicknesses during galvanostatic cathodic reduction assuming the optical constant of the oxide $\left(n_{F}-k_{F}\right)$ to be in the range 1-3.5 and 0-1 respectively. Film formation and reduction conditions are the same as for figure 2.]

trois (Fig. 6) parmi toutes les solutions possibles, les indices caractérisant le film étant :

$$
\begin{aligned}
& n_{\mathrm{F}}^{*}=1,47-0,80 i \\
& n_{\mathrm{F}}^{*}=1,70-0,40 i \\
& n_{\mathrm{F}}^{*}=1,66-0,25 i
\end{aligned}
$$

Ce résultat concorde d'ailleurs avec les calculs de Mac Crackin [27] : ce dernier a montré qu'un tel processus s'avérait faux pour des épaisseurs de film absorbant inférieures à $100 \AA$ lorsque $\Delta_{\text {mesure }}$ et $\psi_{\text {mesure }}$ étaient respectivement entachés d'erreur expérimentale de $0,04^{\circ}$ et $0,02^{\circ}$ ce qui est notre cas. Or ces erreurs absolues sont tout à fait courantes pour des mesures effectuées in situ sur des surfaces immergées et il est pratiquement impossible de les réduire.

Ainsi, les deux approches pour rendre l'ellipsométrie self-consistante - variation de l'angle d'incidence et variation de l'épaisseur du film - se conçoivent peut-être dans le cas d'épaisseurs supérieures ou au 
moins égales à $100 \AA$, à condition toutefois d'avoir vérifié auparavant l'homogénéité des différents films utilisés. Ce dernier point n'est pas toujours facile à réaliser, surtout lorsqu'il s'agit de films formés in situ.

c) Changement du milieu environnant. - Des mesures ellipsométriques peuvent être effectuées sur un seul film en contact avec différents milieux environnants d'indice de réfraction connu. Théoriquement ceci est possible si l'indice de réfraction du film ne varie pas par suite du changement du milieu environnant. Il y a cependant tout lieu de craindre une telle évolution du fait :

- soit d'une absorption possible par le film de la solution constituant le nouveau milieu,

- soit d'une dissolution de ce film,

- soit d'un dépôt d'un nouveau film sur celui déjà existant et faisant l'objet de l'étude. Si ces différents effets n'intervenaient pas, l'indice de réfraction pourrait être calculé à condition que l'épaisseur du film demeure constante lors du changement du milieu environnant, ce qui n'est pas prouvé [28, 29, 30].

En conclusion, cette approche semble également à déconseiller dans le cas d'étude de films très minces absorbants où il est difficile d'exclure la formation d'une couche superficielle dont l'indice optique serait voisin de celui de la solution. Nous avons en effet récemment mis en évidence une variation importante de l'indice optique d'un film passif de fer formé dans une solution contenant ou non de l'arsenic [12] : $n_{\mathrm{F}}^{*}$ est passé de 2,4-0,4 $i$ pour un film formé en solution neutre exempte d'arsenic à $n_{\mathrm{F}}^{*}=1,47-0,80 i$ lorsque celui-ci était formé dans la même solution en présence d'arsenic. La même remarque a été effectuée par Dell'Oca et Young [4] qui ont observé une valeur plus faible de l'indice optique d'un film anodique de tantale formé dans une solution diluée d'acide phosphorique ; ils ont attribué cette valeur à une incorporation de phosphate.

d) Changement de substrat. - Des mesures pourraient être effectuées sur un même film déposé sur différents substrats d'indice de réfraction connu. Ceci peut s'appliquer à des films épais (200 à $2000 \AA$ ) facilement détachables, mais ne peut être envisagé dans le cas de films très minces $(d<50 \AA)$. Ainsi, même par un plus grand nombre de mesures indépendantes, il semble difficile de rendre quantitative i'ellipsométrie lorsqu'elle est appliquée à l'étude d'un film mince, absorbant, homogène. Une autre possibilité à envisager est alors d'essayer de lever l'indétermination par une mesure des constantes optiques du film ou de son épaisseur.

4.1.2.2. Détermination préalable des constantes optiques du film. - Si ce procédé se conçoit pour des films épais, il semble difficilement appliquable à des films très minces formés in situ dans la mesure où le changement d'environnement modifierait la couche initialement étudiée. En outre retenir pour indice optique du film étudié celui déterminé sur un film équivalent mais formé par un mécanisme différent (oxyde anodique-oxyde thermique) demande également une certaine prudence, en particulier pour l'étude de films très minces. Donc, là encore, cette approche ne semble pas satisfaisante. Est-il plus raisonnable de déterminer l'épaisseur du film ?

4.1.2.3. Détermination préalable de l'épaisseur d'un film mince absorbant. - Dans le cas de films anodiques formés en solution aqueuse, de nombreux auteurs déduisent l'épaisseur du film des mesures coulométriques $[8,9,31]$. Une telle détermination présente à notre sens certaines incertitudes : il y a en effet lieu de corriger la charge totale passant à travers le circuit de celle nécessaire à l'oxydation de l'hydrogène adsorbé, ainsi que celle correspondant au métal dissous - généralement déterminée par mesures spectrophotométriques.

Certains auteurs prennent ces corrections en considération, d'autres l'une d'entre elles seulement. Ces incertitudes coulométriques sont résumées sur la figure 7 qui mentionne l'ensemble des résultats établis

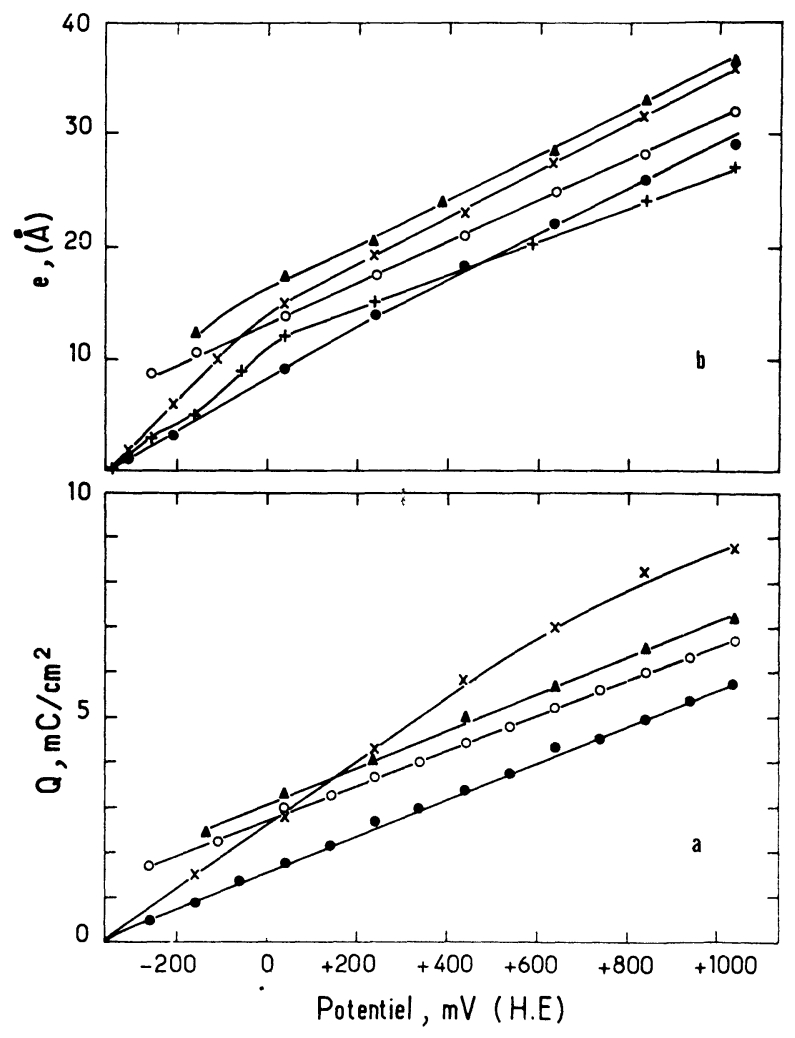

FIG. 7. - Comparaison à partir des données de la littérature, entre les différentes valeurs coulométriques $(a)$ et les épaisseurs déduites (b) : O Nagayama et al. [18] ; $\Delta$ Sato et al. [7]; + Frankenthal [3] ; • Bockris et al. [8] (épaisseur évaluée à partir de $Q_{\text {cath }}$ corrigée) ; × Bockris et al. [8] (épaisseur évaluée à partir de $Q_{\text {cath }}$ total).

[Comparison of thicknesses deduced from coulometric results O Nagayama et al. [18] ; $\Delta$ Sato et al. [7] ; + Frankenthal [31]; $\bullet$ Bockris et al. [8] (thickness deduced from $Q_{\text {cath }}$ corrected) ; $\times$ Bockris et al. [8] (thickness deduced from $Q_{\text {cath }}$ total).] 
dans la littérature, relatifs au système fer-solution neutre de borate de sodium-acide borique étudié par coulométrie. Une variation relative de $40 \%$ est observée sur les mesures coulométriques (Fig. 7a). En outre, la transformation du nombre de coulombs supposés parfaitement définis en nombre d'atomes d'oxygène équivalents puis en épaisseur géométrique équivalente impose la connaissance de la stoechiométrie du composé formé, ainsi que celle du rendement faradique d'oxydation anodique. Il faut noter que ces deux grandeurs sont également indispensables pour la déduction de l'épaisseur géométrique du film à partir du nombre d'atomes d'oxygène directement mesurés par microanalyse nucléaire [12-32]. La figure $7 b$ représente les épaisseurs géométriques d'oxyde de fer passifs investis par coulométrie : l'épaisseur de l'oxyde formé à $+1040 \mathrm{mV}$ (H. E.) varie de $26 \AA$ à $38 \AA$, soit une variation relative d'environ $40 \%$. Ceci est important et ne permettra de retenir une valeur de l'épaisseur qu'avec un maximum de précautions. En effet, en se référant à nos mesures, une épaisseur moyenne de $34 \pm 4 \AA$ entraîne un indice de film variant entre 2,82 et 2,51 alors que le coefficient d'absorption varie entre 0 et 0,06 . Enfin il faut préciser que dans le cas de films passifs formés en solution acide, la coulométrie est difficilement utilisable contrairement au dosage par microanalyse nucléaire [28-29].

4.2 Conclusion. - Ce paragraphe illustre donc la grande difficulté d'arriver à une valeur univoque pour la détermination de l'indice de réfraction d'un film absorbant très mince. Même la connaissance de l'épaisseur renforce cette conclusion dans la mesure où l'indice optique ne sera jamais déduit avec une précision suffisante pour pouvoir définitivement caractériser la structure du film étudié. Ainsi, l'investigation par ellipsométrie d'un film absorbant très mince de structure inconnue n'apportera aucune information caractérisant les propriétés optiques du film mais seulement un ordre de grandeur de l'épaisseur dans les cas les plus favorables. Par contre, lorsque l'homogénéité du film, sa stoechiométrie, et son épaisseur sont par ailleurs déterminées, l'ellipsométrie est alors le complément permettant de déduire des informations précises sur l'indice optique, donc sur la structure de ce film.

5. Etude ellipsométrique de films minces, homogènes, compacts et d'épaisseur connue : résultats et interprétation. - En combinant les études nucléaires et ellipsométriques, nous pouvons choisir parmi toutes les solutions ellipsométriques possibles celle dont le couple $n_{\mathrm{F}}, k_{\mathrm{F}}$ fournira la même épaisseur que l'épaisseur nucléaire équivalente et rigoureusement déterminée précédemment (paragraphe 3 ). Nous obtenons alors (Fig. 8) la valeur suivante de l'indice optique

$$
n_{\mathrm{F}}^{*}=(1,47 \pm 0,08)-(0,80 \pm 0,05) i \text {. }
$$

Les valeurs obtenues pour $n_{\mathrm{F}}^{*}$ impliquent deux remarques :

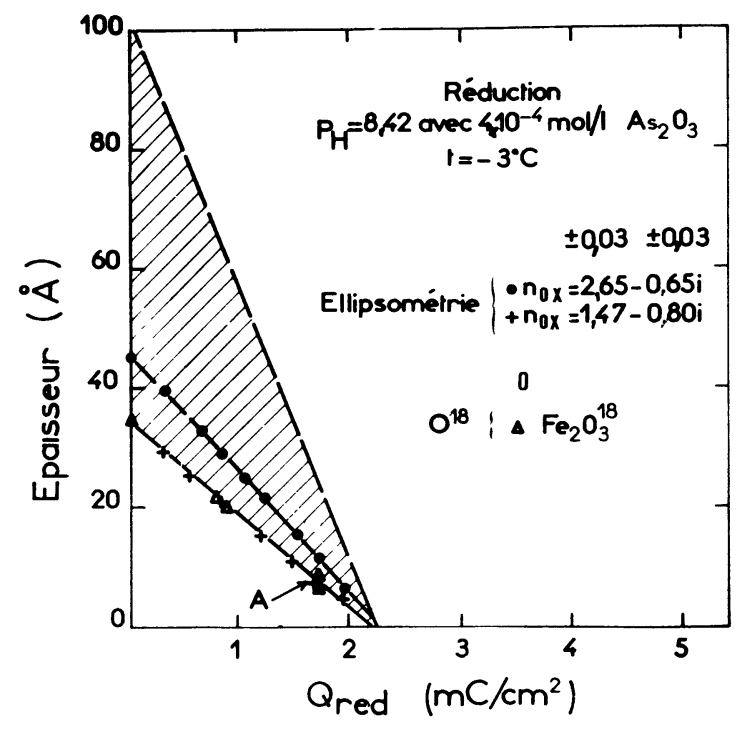

Fig. 8. - Variations de l'épaisseur lors de la réduction cathodique à $10 \mu \mathrm{A} / \mathrm{cm}^{2}$ d'un film passif de fer formé potentiostatiquement en 3 min. $\left(I_{\text {final }}=10 \mu \mathrm{A} / \mathrm{cm}^{2}\right)$ à $+940 \mathrm{mV}$ dans une solution neutre de $0,15 \mathrm{~N}$ de borate de $\mathrm{Na}$ et d'acide borique contenant $4 \times 10^{-4} \mathrm{M} /$ litre d'As ${ }_{2} \mathrm{O}_{3}$ et maintenu à $-3^{\circ} \mathrm{C}$. $\triangle$, épaisseurs nucléaires équivalentes de films passifs déduites des quantités d'O ${ }^{18}$ et d'As. Zone hachurée, épaisseurs de films calculées à partir de données ellipsométriques obtenues lors de la formation de films passifs lorsque $n_{\mathrm{F}}$ et $k_{\mathrm{F}}$ sont supposés varier dans les intervalles $1-3,5$ et $0-1$.

[Change of thickness of iron during galvanostatic cathodic reduction at $10 \mu \mathrm{A} / \mathrm{cm}^{2}$ for the passive film formed potentiostatically at $+940 \mathrm{mV}$ in $0.15 \mathrm{~N}$ boric-borate buffer solution at $\mathrm{pH}=8.42$ containing $4 \times 10^{-4} \mathrm{M} /$ liter $\mathrm{As}_{2} \mathrm{O}_{3}$ as inhibitor and maintened at $-3^{\circ} \mathrm{C}$. Shaded zone, film thicknesses calculated from ellipsometric data obtained during cathodic reduction of the film assuming the optical constant of the oxide $\left(n_{\mathrm{F}-} k_{\mathrm{F}}\right)$ to be in the range 1-3.5 and $0-1$ respectively. $\Delta$ are the equivalent nuclear thicknesses of passive films deduced from the $18 \mathrm{O}$ and As constants.

1) Les erreurs entachant $n_{\mathrm{F}}$ et $k_{\mathrm{F}}$ sont élevées, respectivement 0,08 et 0,05 . Ces erreurs sont directement reliées à l'indétermination $(5 \%)$ de l'épaisseur nucléaire [20]. Cette imprécision importante sur la valeur de l'indice optique ne permettra pas de déduire des informations sur l'homogénéité du film. Nous avons vu en effet que cette dernière était reliée à la constance de l'indice optique, lequel est au plus entaché d'une imprécision égale à 0,008 , provenant de l'erreur expérimentale commise sur $\Delta$ et $\psi\left(\delta \Delta=\delta \psi=0,03^{\circ}\right)$. Aussi une détermination de $n_{\mathrm{F}}$ et $k_{\mathrm{F}}$ avec une erreur largement supérieure à 0,008 ne donnera pas d'informations relatives à la structure du film; ces dernières devront être mises en évidence par une méthode complémentaire. Dans le cas du système présentement décrit, la microanalyse nucléaire a permis de répondre à cette question.

2) La deuxième remarque est relative à l'influence de la rugosité sur la détermination de $n_{\mathrm{F}}$ et $k_{\mathrm{F}}$. Nous avons précédemment été amenés à conclure que $\Delta$ et $\psi$ variaient avec la rugosité [12] ; ceci a d'ailleurs été 
très récemment confirmé [33] par des études ellipsométriques effectuées sur des substrats de silicium et de germanium où l'effet de la rugosité de surface sur l'état de polarisation de la lumière réfléchie a été observé. Le problème se pose cependant de savoir de quelle façon cet effet de rugosité intervient sur la variation correspondante de $\Delta$ et $\psi$. Une réponse précise est présentement difficile à donner, mais des expériences faisant intervenir différents états de la surface d'un échantillon de fer étudié sous différents angles d'incidence sont actuellement en cours et doivent contribuer à une meilleure compréhension de ce problème.

6. Conclusion. - Notre étude a essentiellement porté sur l'investigation par ellipsométrie de films minces absorbants et homogènes. Nous nous sommes tout d'abord attachés à l'obtention et à la caractérisation de telles couches pour insister ensuite sur la difficulté qu'il y avait à déterminer des valeurs univoques susceptibles de caractériser leur indice de réfraction. C'est ainsi que nous avons montré que l'augmentation du nombre de mesures ellipsométriques, indépendantes les unes des autres, sert seulement à renforcer cette conclusion. Nous pouvions espérer contour- ner le problème en déterminant l'épaisseur : malheureusement la difficulté demeurera lorsque l'épaisseur est calculée à partir d'hypothèses physiques prêtant à discussion. La seule solution envisageable est alors de travailler dans un domaine de longueurs d'onde où l'indice d'extinction $k_{\mathrm{F}} \mathrm{s}$ 'annule. Alors l'ellipsométrie permettra de déterminer l'épaisseur du film, ainsi que son indice de réfraction à la longueur d'onde utilisée. De telles études sont en cours, pour des longueurs d'onde $\lambda$ et de films d'épaisseur $d$ tels que les conditions $\frac{2 \pi n d}{\lambda} \ll 1$ et $\frac{2 \pi k d}{\lambda} \ll 1$ soient respectées.

En dépit de ces difficultés, l'ellipsométrie demeure un outil d'investigation privilégié pour détecter des changements de structure de ces films minces surtout si ces changements ont lieu in situ et pour déterminer l'ordre de grandeur de leur épaisseur. De plus, nous avons montré qu'en plus d'un outil analytique sensible et qualitatif, l'ellipsométrie, combinée à une autre méthode expérimentale, pouvait devenir quantitative lorsqu'elle est appliquée à l'étude de films minces absorbants, si ces derniers sont cependant homogènes et de composition déterminée. Ceci nous aura permis de définir la structure des films passifs qui jouent un rôle fondamental en corrosion aqueuse.

\section{Bibliographie}

[1] ARCHER, R. J., dans Ellipsometry in the Measurement of Surfaces and Thin Films. Eds. E. Passaglia, R. P. Stromberg and J. Kruger, Natl. Bur. Std. Misc. Publ. 256, U. S. Govt. Printing Office Washington (1964) p. 255.

[2] Melmed, A. J. and Carroll, J. J., J. Vac. Sci. Technol. 10 (1973) 164.

[3] Young, L. and Zobel, F. G. R., J. Electrochem. Soc. 113 (1966) 277.

[4] Dell'Oca, C. J. and Young, L., J. Electrochem. Soc. 117 (1970) 1545.

[5] Kruger, J. and Calvert, J. P., J. Electrochem. Soc. 114 (1967) 43.

[6] ORD, J. L. and SMET, D. J., J. Electrochem. Soc. 118 (1971) 206.

[7] Sato, N. and Kudo, K., Electrochim. Acta 16 (1971) 447.

[8] O,M Bockris, J., GenshaW, M. A., Brusic, V. and WroBLAWA. H., Electrochim. Acta 16 (1971) 1859.

[9] McBee, C. L. and Kruger, J., Surf. Sci. 16 (1969) 340.

[10] McCracking, F. L., Passaglia, E., Stromberg, R. R. and SteINBERG, K. L., J. Res. Nat. Bur. Std. 67A (1963) 363.

[11] McCrackin, F. L., J. Opt. Soc. Am. 60 (1970) 57.

[12] AgIUs, B. and SiejKA, J., J. Electrochem. Soc. 122 (1975) 723.

[13] Agius, B. and SiejkA, J., J. Electrochem. Soc. 120 (1973) 1019.

[14] Amsel, G. and Samuel, D., Anal. Chim. 39 (1967) 1689.

[15] Amsel, G., Nadai, J. P., D'Artemare, E., David, D., GIRARD, E. and Moulin, J., Nucl. Instrum. Methods 92 (1971) 481

[16] Amsel, G., dans Physics of Electrolytes. Vol. 1, J. Hladik Editor, p. 127 (Academic Press, London) 1972.
[17] Amsel, G., Cherki, C., Croset, M., Feuillade, G., Nadai, J. P., Ortega, C., Rigo, S. and Siejka, J., Collection Czech. Chim. Commun. 36 (1971) 883.

[18] Nagayama, H. and Cohen, M., J. Electrochem. Soc. 109 (1962) $781 ; 110$ (1963) 670.

[19] McCrackin, F. L., Nat. Bur. Std. Tech., Note (1969) 479.

[20] Agius, B., Thèse, Paris (1975).

[21] Kucirex, J. and Melmed, A. J., J. Opt. Soc. Am. 65 (1975) 611.

[22] Azzam, R. M. A., Zaghloul, A. R. M. and Bashara, N. M., J. Opt. Soc. Am. 65 (1975) 252.

[23] Bashara, N. M. and AzZam, R. M. A., J. Vac. Sci. Technol. 12 (1975) 887.

[24] Gottesfeld, S., Babai, M. and Reichman, B., Proceeding of the Third International Conference on Ellipsometru, p. 36a-36d, Nebraska, 1975.

[25] Drude, Weideman, P., Ann. Phys. 36 (1889) 884.

[26] SChueler, D. G., Surf. Sci. 16 (1969) 104.

[27] McCrakin, F. L., Ellipsometry in the Measurement of Surfaces and Thin Films, Symposium Proceedings, Washington, 1963.

[28] SiejKa, J., Cherki, C. and Yahalom, J., Electrochem. Acta 17 (1972) 2371

[29] Siejka, J., Cherki, C. and Yahalom, J., J. Electrochem. Soc. 119 (1972) 991.

[30] Agrus, B. and Siejka, J., Proceeding of the Fifth International Congress on Metallic Corrosion, p. 228-231, Copyright by N. A. C. E. (1974).

[31] Frankenthal, R. P., Electrochim. Acta 16 (1971) 1845.

[32] Siejka, J., Nadai, J. P. and AmSel, G., J. Electrochem. Soc. 118 (1971) 727.

[33] BrudZewski, K., Appl. Opt. 15 (1976) 115. 